\title{
EVALUACION DEL POTENCIAL NATURAL DE LOS PAISAJES PARA LA ACTIVIDAD AGROPECUARIA EN EL EJEMPLO DEL MUNICIPIO YAGUAJAY, (PROVINCIA SANCTI-SPIRITUS, CUBA)
}

\author{
Sara ARCEO y Eduardo SALINAS \\ Universidad de La Habana (Cuba)
}

\begin{abstract}
Resumen: En el presente artículo se pretenden mostrar las ventajas de la concepción integradora del paisaje y la determinación de sus potenciales naturales en la planificación territorial a escala media en Cuba.
\end{abstract}

Palabras clave: Potencial natural, planificación, paisaje integrado.

\begin{abstract}
This article tries to show the advantages of the studies of integrated landscape and the determination of their natural resources in territorial management in Cuba.
\end{abstract}

Key words: Natural resources, management, integrated landscape

\section{INTRODUCCION}

La planificación en relación con el uso de la tierra es de origen antiguo. Las civilizaciones de Mesopotamia, China, Egipto y Perú, entre otras, requirieron de un alto grado de organización y planificación con vistas a construir, operar y mantener sistemas de irrigación, los que constituyeron la base de su agricultura.

En los países en vías de desarrollo la planificación para el uso agropecuario debe servir a la toma de decisiones en el desarrollo socio-económico del campo, con el interés de los requerimientos nacionales, es decir, establecer el uso racional de sus recursos naturales y prevenir la degradación ambiental como consecuencia de un incremento de la producción y de la necesidad del desarrollo acelerado. 
Las últimas décadas han conocido de un intenso interés en los estudios integrales como parte de los enfoques multidisciplinarios aplicados al análisis y evaluación de la compleja interrelación que se establece entre la naturaleza y la sociedad y que en la actualidad se ha visto llevada al plano público y político, dado por la destrucción y degradación acelerada a que están siendo sometidos extensos territorios de nuestro planeta.

Los componentes naturales del paisaje considerados como factores activos y no como simples elementos, junto a los económicos, sociales y culturales deben ser tomados en consideración e integrados en la planificación regional.

Pretendemos, en forma resumida, mostrar las posibilidades y ventajas de la concepción integradora del paisaje y la determinación de sus potenciales naturales en la planificación territorial a escala media, en las condiciones físico-geográficas y socio-económicas particulares de Cuba y como parte de los estudios encaminados a la confección de los planes directores para el desarrollo de los municipios a corto y mediano plazo.

\section{POSICION GEOGRAFICA Y PARTICULARIDADES NATURALES DEL TERRITORIO}

El municipio Yaguajay con una extensión de $1.032,28 \mathrm{~km}^{2}$ y más de 58.000 habitantes se encuentra situado al norte de la provincia Sancti-Spíritus en la porción central de la isla de Cuba, (Fig. 1) en un área donde predominan las estructuras geológicas orientadas sublatitudinalmente con una gran complejidad tectónica y litológica, lo que es característico para todo el distrito de Cuba Central y que influye de manera significativa en el relieve del municipio.

$\mathrm{Al}$ norte encontramos una extensa área llana fluvio-marina, deltaica y marina, con predominio de ascensos neotectónicos débiles o descensos relativos con diversos niveles que van desde una llanura muy baja pantanosa $(1 \mathrm{~m})$ hasta una llanura alta de más de $60 \mathrm{~m}$ de altura, desarrolladas todas sobre rocas de la cobertera de la plataforma moderna de edad Pleistoceno-Holoceno representada por depósitos carbonatados, terrígenos y turbosos, arcillas, arenas, gravas y calcarenitas entre otras; con algunos afloramientos de grauvacas, brechas, tobas, andesitas y basaltos del Eoceno. En general esta llanura presenta pendientes menores de $2^{\circ}$, una disección baja y el predominio de los procesos acumulativos. 


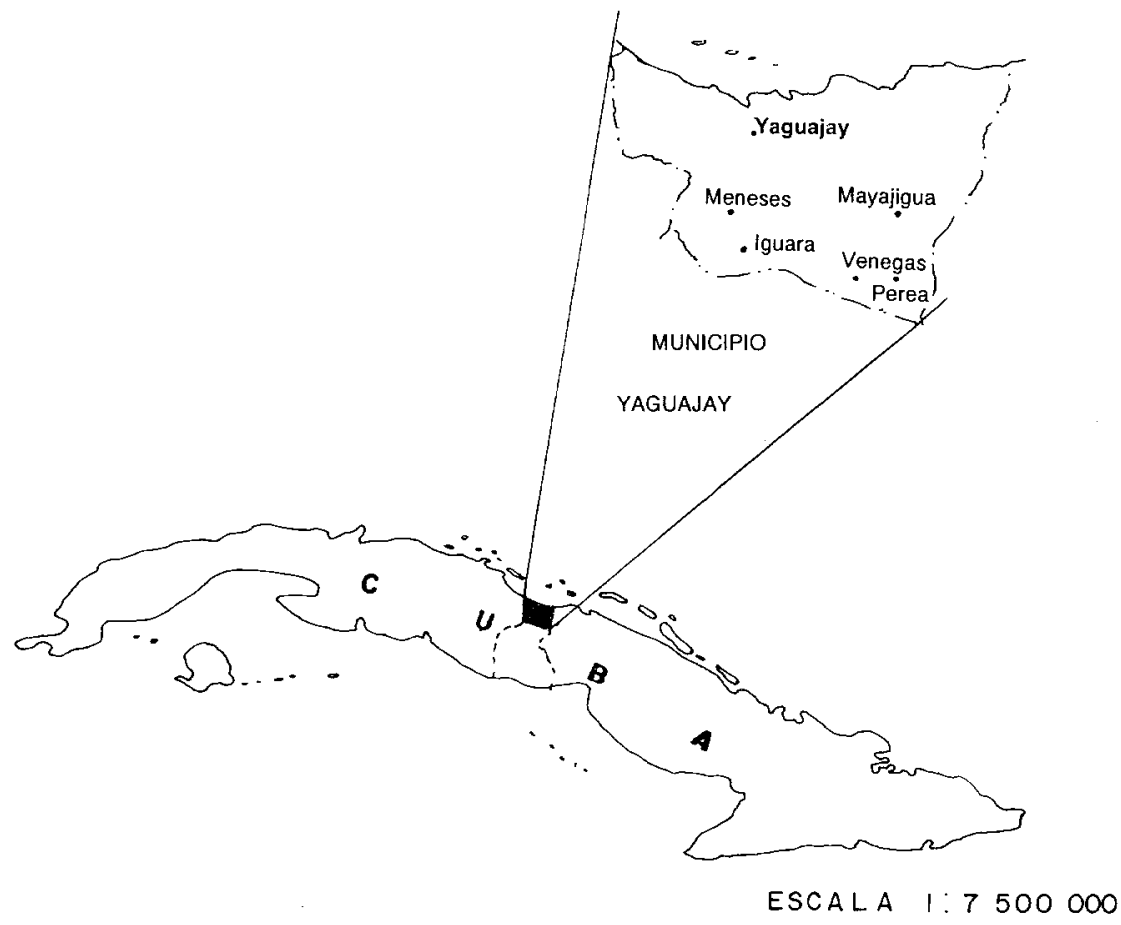

Figura 1.- Situación del municipio Yaguajay en el territorio nacional.

Al sur de esta llanura y al centro del municipio se localiza un área de alturas tectónico-estructurales, de landschaft y bloque entre 200 y $400 \mathrm{~m}$, con predominio de ascensos neotectónicos moderados o intensos, compuesta por rocas pertenecientes a las cuencas superpuestas sobre el arco volcánico cretácico de molasa superior carbonatada terrígeno-detrítica, cubierta por brechas, conglomerados, esquistos, tobas, etc. del Eoceno y por estructuras de los manto-plegamientos alpinos, formadas por un complejo carbonatado y carbonatado-silíceo. Estas alturas presentan pendientes medias a fuertes con una disección alta y el predominio de los procesos denudativos y cársicodenudativos.

El límite meridional de las alturas está constituido por una depresión tectónica con dirección este-oeste, ocupada actualmente por los valles de los ríos Jatibonico del Norte y Caunao que han cubierto con potentes espesores de sedimentos, las rocas más antiguas. Las pendientes son de ligeras a medias con una fuerte disección y el predominio de los procesos fluviales. 
El extremo sur del municipio está ocupado por una alineación de alturas bajas $(100 \mathrm{~m})$, constituidas también por manto-plegamientos alpinos, compuestos por rocas de la asociación afiolítica (serpentinitas, peridotitas, etc.). Aquí encontramos pendientes fuertes, una disección media y el predominio de los procesos denudativos.

El clima presenta diferencias claras de norte a sur en relación directa con la elevación del relieve y la distancia al mar, la parte norte del municipio se puede considerar como muy cálida y subhúmeda con temperaturas medias anuales entre $24 \mathrm{y}$ $26^{\circ} \mathrm{C}$, precipitaciones inferiores a $\operatorname{los} 1.400 \mathrm{~mm}$ anuales y una humedad relativa de 85-90 \%; la zona de alturas presenta un clima cálido y húmedo, subtropical en invierno (Lecha, L. y Florida, A. 1989), con temperaturas medias anuales entre 22 y $24^{\circ} \mathrm{C}$ (media de invierno inferior a $18^{\circ} \mathrm{C}$ ), precipitaciones superiores a los $1.600 \mathrm{~mm}$ y una humedad relativa de más del $95 \%$.

El escurrimiento superficial al norte es bajo e inferior a los $400 \mathrm{~mm}$, al centro y sur del municipio sobrepasan los $500 \mathrm{~mm}$, además se presentan problemas de empantanamiento y mal drenaje en extensas áreas de la llanura norte.

Los suelos predominantes son los pardos, con o sin carbonatos, oscuros plásticos gleysados, hidromórficos, aluviales, poco desarrollados y, en menor medida, los ferralíticos rojos y fersialíticos.

La vegetación originaria, formada por diversos tipos de bosques y matorrales, ha sido fuertemente modificada y sustituida por cultivos o vegetación secundaria, encontrándose sólo en las alturas cársicas, al centro, restos de bosque semideciduo y vegetación de mogote, en Cayo Caguanes (matorral xeromorfo costero), en las costas bajas el manglar y en las alturas de serpentinitas el matorral xerofítico muy degradado por la acción del fuego y el pastoreo extensivo.

\section{PAISAJES}

El término paisaje, usado en la literatura científica desde el siglo pasado, ha alcanzado una connotación superior en los últimos tiempos por un uso público y político mayor, condicionado esto por la destrucción y degradación acelerada a que están siendo sometidos extensos territorios de nuestro planeta.

El paisaje geográfico, geosistema, complejo territorial natural, unidad ambiental (landschaft según los alemanes) debe ser considerado como una unidad espacial integral, objetivamente existente, homogénea en sus diferentes partes, que se 
encuentra en un estado constante de intercambio de sustancia y energía, estando constituida por la asociación dialéctica de componentes, formados bajo la influencia de los procesos naturales y la actividad modificadora del hombre. Ellos se pueden considerar como medio de vida y de satisfacción de las necesidades culturales, estéticas y recreativas del hombre y portador de recursos naturales y como espacio para el desarrollo socio-económico de la humanidad.

La situación del municipio en los límites de dos regiones físico-geográficas, con características muy diferentes de litología, relieve y humedecimiento, condicionan una complejidad alta de los paisajes que se evidencia en la existencia de 10 localidades y 43 comarcas (Fig. 2).

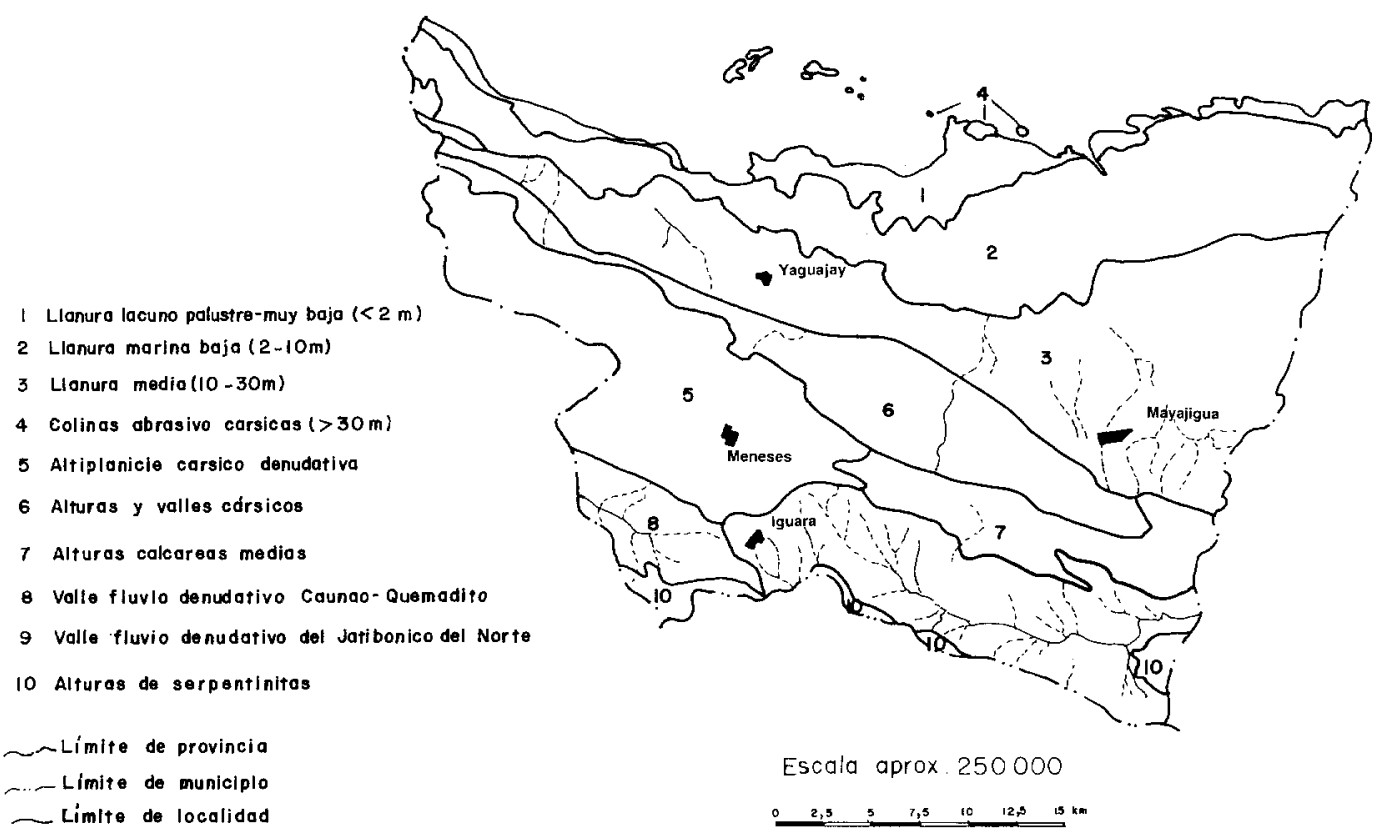

Figura 2.- Mapa de complejos territoriales naturales. 
Es característico para este territorio la influencia que ejercen en la formación y desarrollo de sus paisajes, la altura y la distancia al mar, ya que éstas contribuyen a modificar las condiciones de humedecimiento y por tanto los procesos de formación del relieve y los suelos.

Se debe destacar además, aunque no ha sido muy valorado en investigaciones anteriores, el factor exposicional en las alturas y los valles interiores, lo que condiciona la existencia de cambios en los suelos y la vegetación como consecuencia de las variaciones en el humedecimiento y la insolación.

Por último, se puede afirmar que el territorio, salvo raras excepciones, sufrió una fuerte modificación de su naturaleza por la asimilación de extensas áreas para la actividad agrícola y ganadera.

\section{POTENCIAL NATURAL DE LOS PAISAJES PARA LA ACTIVIDAD AGROPECUARIA}

El concepto de potencial del paisaje como parte de una amplia concepción de la síntesis del paisaje, ha sido desarrollado de una forma destacada por los geógrafos alemanes de la llamada "Escuela del Potencial" (Neef, Hoose y Mannsfeld) y por los checos (Mazur, Drdos, Huba, Othael, entre otros). Además ha sido empleado por algunos geógrafos polacos y presenta semejanza con el método de planificación ecológica de los franceses. (Falque, N. 1972 y Tricart y Killian, 1982).

En la Geografía actual se extiende el término de potencial del paisaje como capacidad productiva de los complejos naturales, según la asociación de determinadas posibilidades y condiciones actuales para determinados tipos de utilización, con el objetivo de satisfacer las necesidades de la sociedad humana, y que refleja el posible cumplimiento por parte del paisaje de determinadas funciones socio-económicas que a él se asignan en dependencia de sus propiedades naturales, por ejemplo: potencial turístico, agrícola, forestal y otros.

En Cuba ha sido utilizada por diversos autores para diferentes tipos de evaluación (Quintela, F.J. 1984, Garcia, E. y otros 1985, Carmona, M. 1985, Arcia, M. y Rodriguez, C. 1985, Salinas, E. 1988, 1989 y Sánchez, C.M. 1986).

Las investigaciones del potencial del paisaje se basan en tres aspectos esenciales: 
- La unidad orgánica del potencial del paisaje con el desarrollo socio-económico de la sociedad, que implican la armonía entre el paisaje y la economía.

- El establecimiento del beneficio social sobre el local, ramal u otro interés parcial.

- El establecimiento de la conservación del potencial reproductivo del paisaje para el futuro.

El desarrollo del concepto de potenciales naturales es, sin duda, una posible evaluación más precisa de la capacidad de una unidad natural bajo las condiciones actuales y futuras de utilización.

El potencial, como categoría científica, corresponde a los recursos naturales potenciales de la economía. Ellos pasan a la categoría de recursos naturales realmente utilizables, como un proceso histórico controlado por la capacidad tecnológica y el desarrollo socio-económico de la sociedad. La principal ventaja de la concepción del potencial del paisaje es el hecho de no estar alterada por aproximaciones sectoriales para la utilización de paisaje, además supera también la concepción del paisaje como un espacio ilimitadamente explotable.

El análisis del material obtenido en el levantamiento de campo de los paisajes y de la información cartográfica y áreas disponibles, posibilitó la confección de una matriz de doble entrada para la evaluación de los paisajes, considerando como indicadores para la misma aquellos aspectos que consideramos tienen una relación estrecha con las posibilidades de utilización de un territorio para la actividad agropecuaria (altimetría, temperatura, precipitación, capacidad agrológica de los suelos, entre otros) o pueden constituirse en limitantes para dicha actividad (salinización, pendiente, drenaje, erosión, etc.).

Se analizaron los criterios de evaluación, anteriormente mencionados, en cada comarca y se les dió valor, 5,3 ó 1, según la magnitud de influencia en la actividad evaluada; por ejemplo, si analizamos la pendiente para la actividad agropecuaria, según rasgos establecidos, si es favorable, toma valor 5, si es media, 3 y si es desfavorable, valor 1 (Fig. 3). 


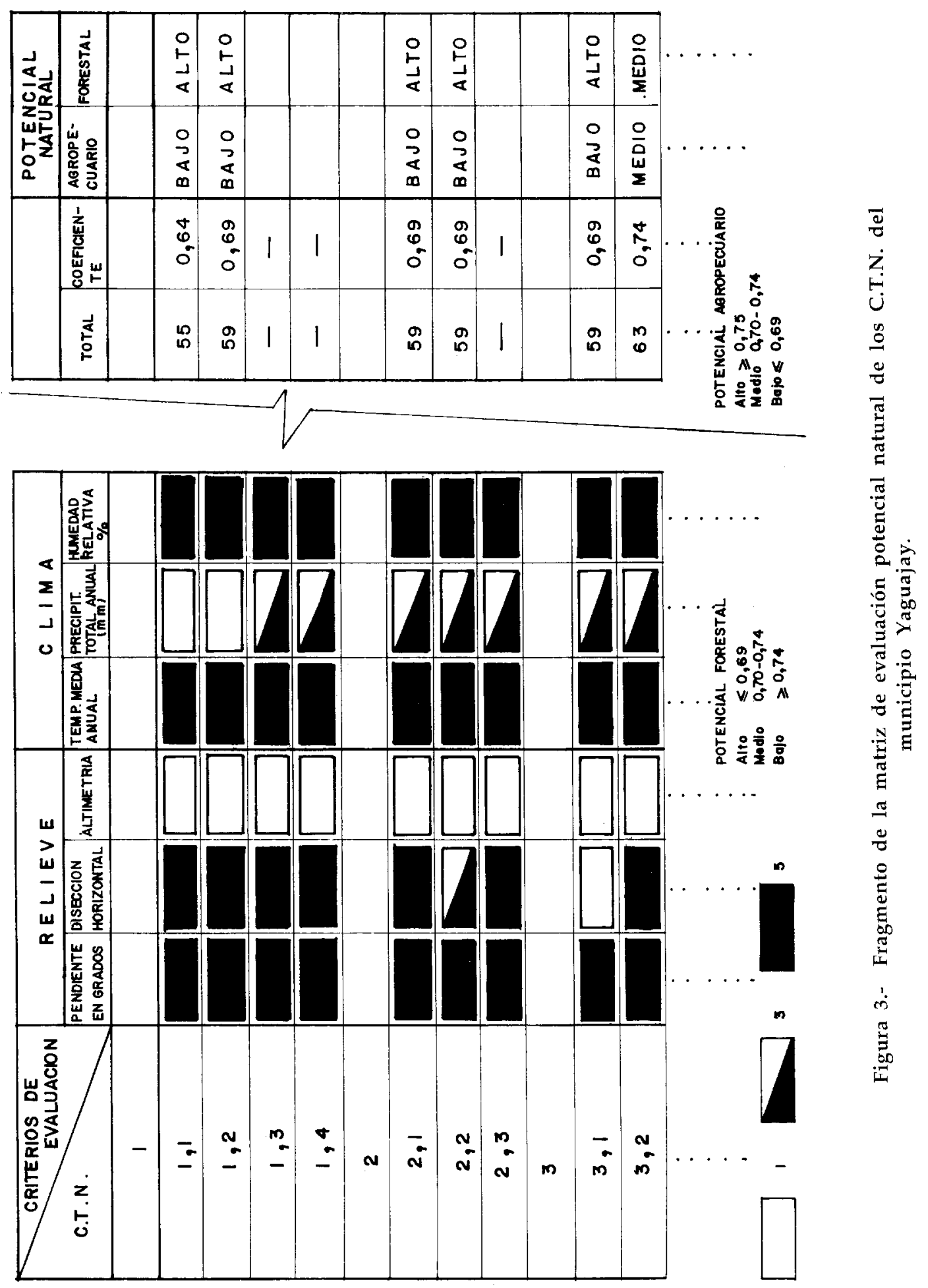


En cada paisaje se obtuvo un valor total de la suma de los valores parciales, el que fue dividido entre el valor máximo posible a obtener, en este caso 85, obteniéndose un coeficiente al que hemos denominado coeficiente de potencial natural y cuyos valores variaron en nuestra área de estudio entre 0.48 y 0.83 con un valor medio de 0.68 de aquí se obtuvieron los intervalos para la clasificación de las unidades lo que sirvió de base para la confección del mapa de potencial agropecuario.

Los valores más altos del potencial agropecuario se localizaron en la altiplanicie y en las terrazas de los ríos Jatibonico del Norte y Caunao-Quemadito, donde existen pocas limitantes para el desarrollo de la agricultura y la ganadería.

Los valores más bajos se corresponden con las superficies llanas cercanas al mar, las colinas residuales, las alturas cársicas y de serpentinitas entre otros, donde se presentan fuertes limitantes para el desarrollo agropecuario como son: salinización, erosión, acidez y mal drenaje de los suelos entre otros (Fig. 5). Por último se analizó la distribución del coeficiente de potencial natural de los paisajes, lo que permitió establecer un agrupamiento de los mismos en función de su valor (Fig. 4).

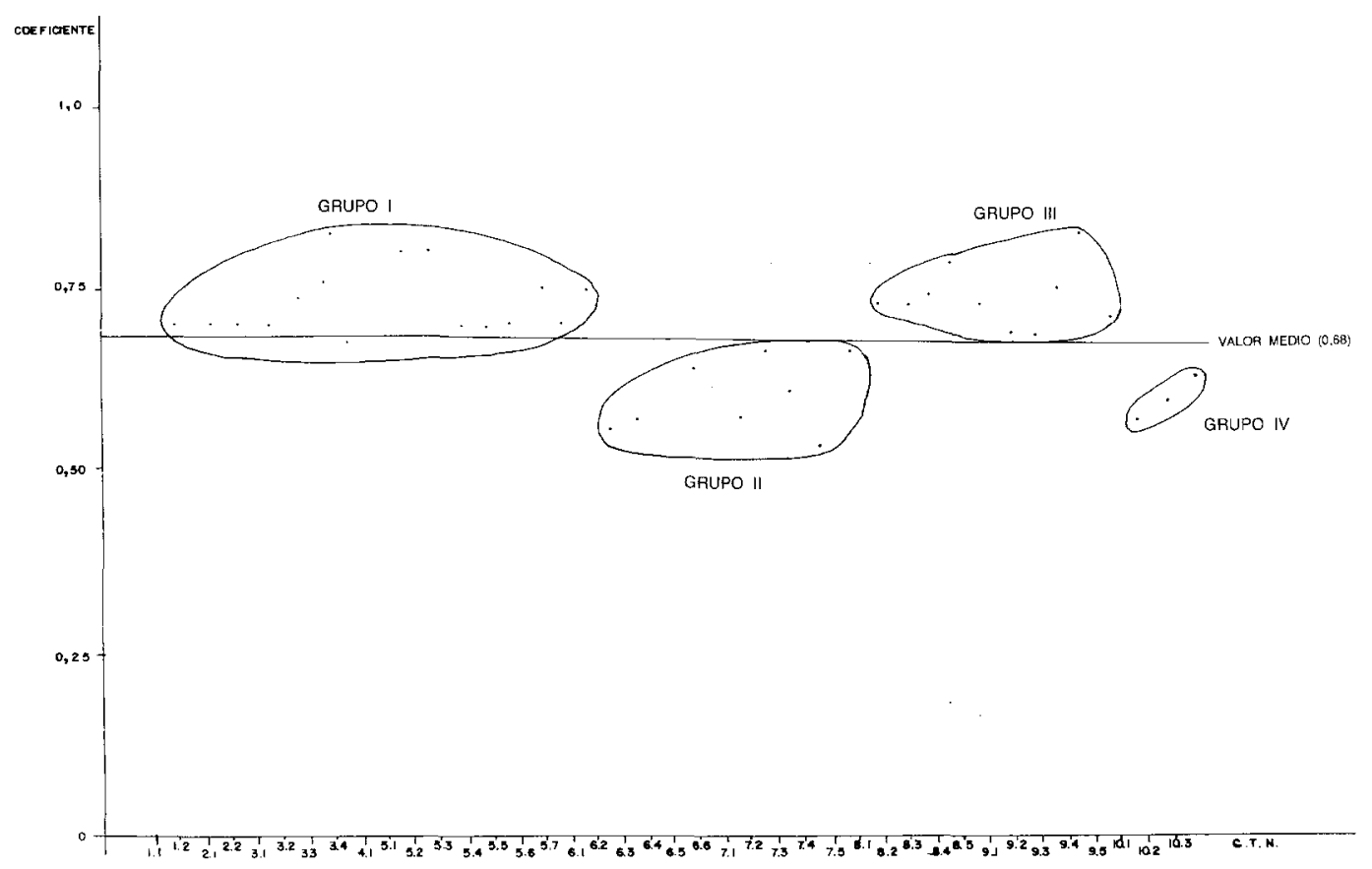

Figura 4.- Distribución del coeficiente de potencial natural para la actividad agropecuaria de los C.T.N. municipio de Yaguajay 


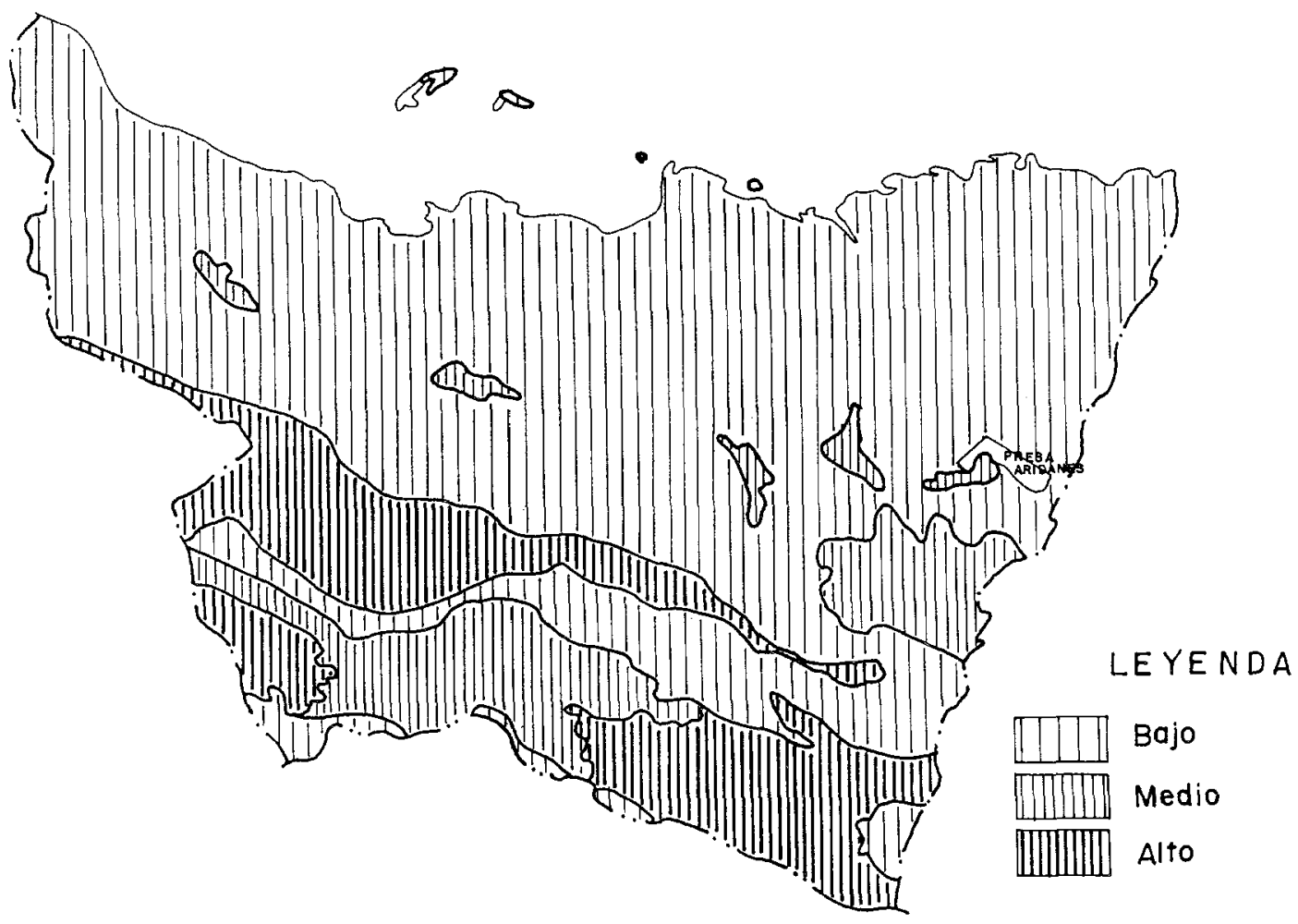

Figura 5.- Mapa de potencial natural agropecuario.

Estos grupos se corresponden bastante bien con las grandes divisiones físicogeográficas del territorio; el grupo I, con la llanura norte y sus diversos niveles, con valores superiores a la media; el grupo II, representado por las alturas cársicodenudativas, con valores muy inferiores a la media; el grupo III, constituido por las llanuras fluviales de los ríos Jatibonico del Norte y Caunao-Quemadito, con valores altos, y por último el grupo IV, representado por las alturas de serpentinitas poco desarrolladas en el territorio del municipio pero con valores muy bajos del potencial para la actividad agropecuaria.

Posteriormente, se analizó la compatibilidad del potencial natural obtenido y el uso actual de los paisajes existiendo dos posibilidades:

- Compatible, cuando el uso del territorio está acorde con el potencial. 


\section{UNIDADES DE PAISAJE}

1.- LLANURA LACUNO-PALUSTRE MUY BAIA (2 m)

1.1. Permanentemente inundada con manglar

1.2. Estacionalmente inundada con pastos y bosque de ciénaga.

2.- LLANURA MARINA BAJA.

2.1. Ocasionalmente inundada con pastos.

3.- LLANURA MEDIA $(10-30 \mathrm{~m})$

3.1. Ligeramente ondulada con caña de azucar.

6.- ALTURAS Y VALLES CARSICOS

6.4. Colinas $(80-100 \mathrm{~m})$ con pastos y restos de bosque semideciduo

6.5. Superficie ligeramente inclunada con bosque semideciduo degradada y pastos.

6.1. Valle intercomtano con caña de azucar frutos menores y pastos.

6.2. Colinas residuales con manigua, pastos y restos de bosques semideciduos

6.3. Escarpe con bosque semideciduo degradado.

7.- ALTURAS CALC AREAS MEDIAS

7.1. Macizo Central y cimas $(300 \mathrm{~m})$ con pastos y restos de bosques semideciduos.

7.2. Macropendiente sur con pastos y restos de bosques degradado

7.4, Colinas con bosque semideciduo degradado

9.- VALLE FLUVIO-DENUDATIVO DEL RIO JATIBONICO DEL NORTE

9.4. Terrazas medias y altas con pastos

9.5. Superficie alta denudativa con pastos

10.- ALTURAS DE SERPENTINITAS

10.2. Superficie andulada colinosa con bosque arbustivo

\section{E Y EN D A}

SUELOS

ב-C= MDROMORFICOS

$\overline{B E=}$ OSCUROS PLASTICOS GEYSADOS

DETIC PARDO CON.CARBONATOS

IIELTIIIII FERRALITICO ROJO

… HUMICO CALCIMORFICO

FEATE FESIALTTICO ROJO PARDUZCO FEROMAGNESIAL

$\Longrightarrow$ ALUVIAL

F.1. POCO DESARROLLADO ESQUELETICO

IUTII CARSO DESNUDO

VEGETACION Y USO DE LA TIERRA

- MANGLAR

* BosQUe en cienaGa

- bosque simideciduo degradado

i bosque en galeria

$\psi$ MatorRal Xerofitico DEgradado

* MatorRales secundarios (manglar)

* caña de azucar

$r$ PASTOS

FRUTOS MENORES xerofitico
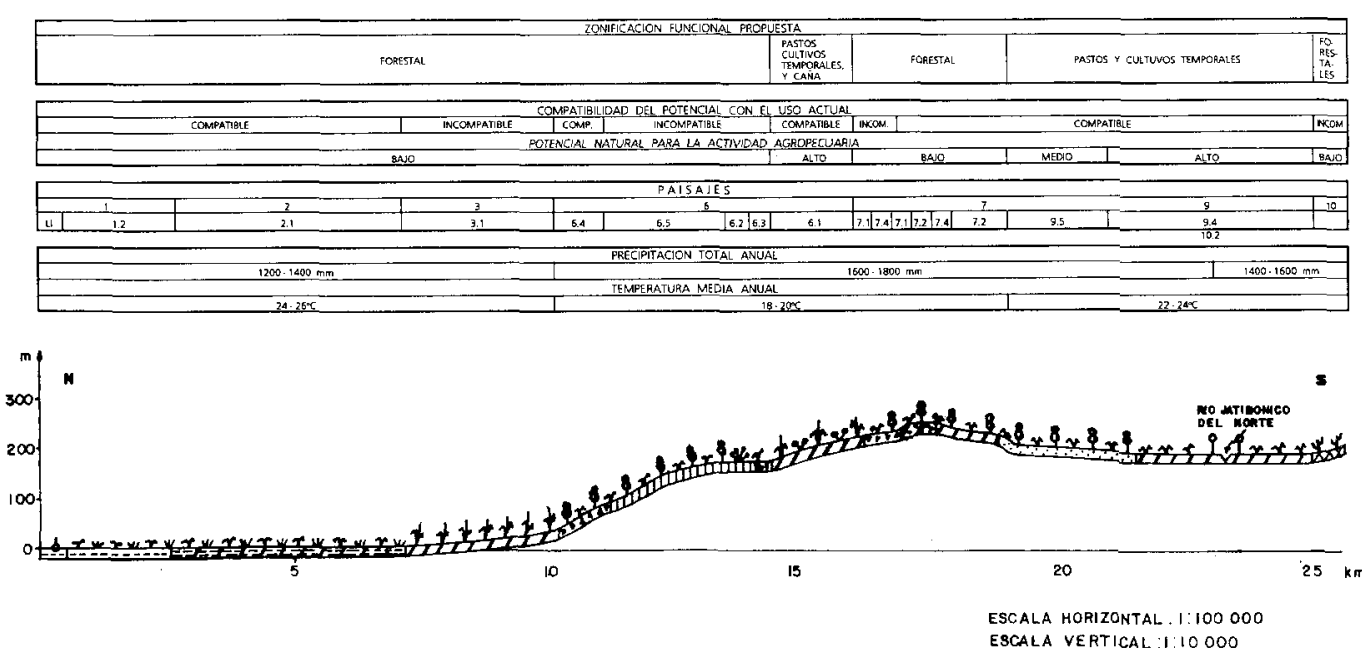

$\sqrt{3.305}$

1

Figura 6.- Perfil físico-geográfico complejo. Municipio Yaguajay. 
- Incompatible, cuando el uso está por encima o por debajo del potencial y se produce o puede producirse una degradación del potencial natural de dicho paisaje.

Este análisis de la incompatibilidad se realizó conjuntamente con la valoración de los factores limitantes lo que permitió proponer un sistema de medidas y recomendaciones para el mejor aprovechamiento del potencial natural de los paisajes (Fig. 6).

\section{CONCLUSIONES}

Las investigaciones del paisaje tienen gran importancia a nivel regional para los planes perspectivos de desarrollo socio-económico, a corto, mediano y largo plazo.

Desde el punto de vista teórico-metodológico y práctico los estudios del paisaje son útiles en la planificación territorial pues permitieron:

1.- Analizar sintéticamente la variada información existente sobre los diferentes componentes y las complejas interacciones que se establecen entre ellos.

2.- Evaluar en forma rápida, confiable y con poco costo, los recursos y condiciones naturales de un determinado territorio.

3.- Establecer el diagnóstico de las acciones transformadoras del hombre sobre los paisajes, lo que permitirá preveer los procesos negativos que ocurrirán a mediano y largo plazo.

Se delimitaron y cartografiaron 10 localidades y 43 comarcas siendo los factores diferenciadores fundamentales de los paisajes las condiciones geológicogeomorfológicas y génesis, para las primeras, y el relieve, suelos, humedecimiento y vegetación, para las comarcas.

La delimitación del potencial natural de los paisajes, utilizando una matriz simple de doble entrada con 17 criterios evaluativos, el relieve, clima, hidrología, suelos, vegetación y valores naturales, permite concluir que el municipio tiene, en general, un potencial bajo para la actividad agropecuaria. 
El análisis de la compatibilidad entre el potencial y el uso actual de los paisajes permitió establecer un sistema de medidas y recomendaciones que a mediano y largo plazo pueden conllevar al uso racional del territorio acorde con sus potencialidades.

La aplicación de los principios teórico-metodológicos planteados en este trabajo, posibilitará la elaboración de planes y programas de desarrollo socioeconómico más objetivos así como contribuirá a optimizar la utilización de los potenciales naturales de los paisajes cubanos, haciendo más efectiva la planificación territorial en sus diferentes niveles.

\section{BIBLIOGRAFIA}

ARCEO, S.M. (1989): Evaluación físico geográfica para el desarrollo agropecuario del municipio de Yaguajay. (Trabajo de Diploma), Facultad de Geografía, U.H. (inédito), 50 p.

D.A.U. Poder popular (1988): Esquema de desarrollo. Municipio Yaguajay. (inédito), sin paginar.

GARCIA, E. y otros (1985): Estudio fisico geográfico del Municipio de Guisa. Provincia Granma para la determinación de sus potenciales naturales. I+P.F. JUCEPLAN, La Habana, 15 p.

HAASE, G. (1978): "Zur Ableitung und Kennzeichnung von Natur Potentialen". Peterm. Geogr. Mitt., 122, 113-125, VEB H. Haack, Gotha, Alemania.

LECHA, L. y LLANES, A. 91988): "Características estacionales de la circulación atmosférica sobre Cuba". Rev. Cubana de Meteorología, Año 1, vol. 1 n 1. 49-56.

MANNSFELD, K. (1979): "Die Beurteilung von Naturranm Potentialen als aufgabe der geographischen Landeschftsforschung". Pett. Geogra. Mitt., 1, 16-27. VED H. Haack, Gotha, Alemania.

MANNSFELD, K. (1986): "Valuation of the natural structure of landscape for planning". Meetinf of I.G.U. Working Group Landscape Synthesis. Landscape Synthesis part. I, 115122.

MATEO, J. , SALINAS, E. y GUZMAN, J.L. (1985): El análisis de los paisajes como fundamento para la planificación de los territorios. Inst. Planif. Física, JUCEPLAN, La Habana, 18 p.

MAZUR, E. y DRDOS, J. (1984): "Conception of Resurces or conception of the handscape potential", En The geographical Research Geograf, cas. 34.4, 305-315, Praga Checoslovaquia. 
NEEF, E. (1984): "Applied Landscape Research ", En Applied Geography and Development, Vol. 24, 38-58, RFA.

PRIEGO, S.A. (1987): Evaluación edafo-morfométrica para un mejor aprovechamiento del recurso tierra en la agricultura para el municipio de Los Palacios.Pinar del Río. (Trabajo de diploma), Facultad de Geografía U.H. (inédito), 70 p.

QUINTELA, F.J. (1984): Determinación de los potenciales naturales para diferentes actividades económicas en la zona de San Antonio de Cabezas, Provincia Matanzas. (Trabajo diploma), Facultad de Geografía U.H. (Inédito), 24 p.

RUBIEKA, M. y MIKLOS, L. (1982): "Landscape-ecological planning (LANDEP) in the process of territorial planning". Ecologia, Vol. 1, 287-312, VEDA Bratislava Checoslovaquia.

SAINI, N,S, y SINHA, R.L.P. (1985): Integrated development planning for a Waterched, School of planning and Arch new Delhi, $152 \mathrm{p}$.

SALINAS, E., MATEO, J. y MAKCESKI, J. (1990): "Problemas de la utilización del potencial de recursos naturales en las CPA (en el ejemplo de la provincia Sancti Spiritus, Cuba). Actas latinoamericanas de Varsovia, Tomo 13, Polonia (en prensa).

SALINAS, E., YANES, L. y ARCEO, S. (1988): "La evaluación agrogeográfica en la planificación regional de Cuba". II Congreso Latinoamericano de Geografia, Montevideo, Uruguay, 15 p.

SALINAS, E., MONTIEL, S. y VALDIVIA, I. (1991): "Yaguajay el área piloto de estudio. Características geográficas generales". Actas Latinoameircanas de Varsovia, Tomo 9, 125138 , Polonia.

SALINAS, E. (1991): Análisis y evaluación de los paisajes en la planificación regional en Cuba. (Tesis para la obtención del Grado Científico de Doctor en Ciencias Geográficas). Universidad de la Habana, 187 p.

SANCHEZ, C.M. (1986): Evaluación de los potenciales naturales de la llanura Norte. Municipio Yaguajay. (Trabajo de Diploma). Fac. de Geografía, U.H. (Inédito), 67 p. 